\title{
Reducing medication errors
}

\author{
The future is near field communication \\ Benjamin Thomas, Hao Shi, Anthony Chow, Julian Surujballi \\ Faculty Reviewer: Shiraz Malik, MD, CCFP (Department of Family Medicine)
}

\section{ABSTRACT}

Hospital readmissions place a heavy burden on healthcare in Canada. Communication errors often occur at vulnerable time points like peridischarge, and these can lead to downstream adverse drug events necessitating rehospitalization. Existing mechanisms to address this problem still have room for improvement. Discharge summaries, for example, may be late, erroneous, or incomplete. Medication reconciliation processes directly integrated with electronic medical records have demonstrated advantages in terms of legibility and timeliness but unfortunately encourage risky behaviours like indiscriminate copy and pasting, leading to new errors of a different sort.

A single unified medication list that could be kept on a patient's person at all times can ensure that medication information is always present at medical appointments and is synchronously updated among all involved practitioners. Near-field communications (NFC) may facilitate the creation and maintenance of such a list. It is a relatively new wireless technology that has advantages over radio-frequency identification and Bluetooth. It allows information to be recorded to and read from objects as small and thin as a sticker. Technologies based on NFC may prove useful for consolidation patient health information into "one source of truth".

\section{INTRODUCTION}

Unintentional hospital readmissions present a significant challenge to the Canadian healthcare system. During an 11-month period in 2012, hospital readmissions constituted $8.5 \%$ of all acute care admissions in Canada and consumed $\$ 1.8$ billion of healthcare spending. ${ }^{1}$ In the United States (US), the problem is no less dramatic, with a hospital readmission rate of $20 \% .^{2}$ Importantly, approximately $23 \%$ of these readmissions were preventable. ${ }^{3}$ Across Canada, the diagnoses associated with the highest readmission rates were heart failure at $21 \%$ and chronic obstructive lung disease (COPD) at $18.8 \%{ }^{1}$ these rates were similar in the United States. ${ }^{4}$

A study based in the US found that $66 \%$ of readmissions within 2 weeks of discharge were due to adverse drug events (ADE), two-thirds of which were deemed to be preventable. ${ }^{5}$ In addition, according to a separate American trial, $19 \%$ of patients experience ADEs postdischarge, $6 \%$ of which were considered serious. ${ }^{6}$

Medication errors that occur during transitions between care settings, such as in the weeks preceding and following discharge (peridischarge), are important and preventable sources of ADE.,8 A review of 1245 inpatient encounters found that just over $50 \%$ of patients had one or more discrepancies between their inpatient and home medications as determined by comprehensive chart review; importantly, the vast majority of these discrepancies had the potential to cause harm. ${ }^{9}$ This suggests that medication errors may be a major cause of preventable hospital readmissions and correspondingly represent a prime target for reducing patient morbidity postdischarge and for decreasing unnecessary healthcare expenditure.

\section{MEDICATION ERRORS}

Medication errors have myriad causes; polypharmacy, decreased patient cognitive ability, and certain categories of medications are specifically implicated in increasing the risk of readmissions. ${ }^{10}$ The class of medication most strongly associated with medical errors is cardiac medications, followed by COPD medications and then blood pressure medications; ${ }^{11}$ these comparative risks are reflected by the readmission rates for their associated medical conditions. ${ }^{1}$

The scope of the problem is large: one review of 22 studies found that up to $67 \%$ of patients had medication discrepancies upon admission to hospital, ${ }^{12}$ while a separate study of 471 patients found that $59.2 \%$ had "a misunderstanding in the indication, dose, or frequency [of] a cardiac medication” after discharge. ${ }^{13}$

Confusion among patients and providers regarding what constitutes an accurate medication list is worsened by frequent medication changes and by having many prescribers involved in care. ${ }^{14}$ Generally, deficiencies in hospital protocols underlie most medical errors that occur during discharge, with failures in communication being the most common reason. ${ }^{6}$ Unsurprisingly, interprofessional communication has become a popular subject of quality improvement initiatives. Some relevant tools and strategies in widespread use today include discharge summaries, medication reconciliation, and computerized order entry and documentation.

\section{DISCHARGE SUMMARIES}

Historically, discharge summaries were necessary only as documentation since the same attending physician usually managed inpatient and outpatient care. On the other hand, modern discharge summaries are relied upon to convey pertinent details, such as primary diagnosis, physical examination, summary of investigations, discharge medications, and follow-up instructions, to community physicians assuming responsibility for care. ${ }^{15}$ Unfortunately, according to a systematic review of 73 research articles investigating discharge summaries, any of the aforementioned sections could be missing, even when explicitly required by institution guidelines. Furthermore, discharge summaries arrived after the discharged patients' first follow-up appointment (or not at all) up to $53 \%$ of the time, ${ }^{14}$ leaving patients to recall and explain the circumstances of 
their inpatient stay and their new management plan.

Discharge summaries continue to be improved upon as vital tools of interprofessional communication. For example, in addition to benefits seen with standardised forms and use of electronic systems, a brief one-time educational workshop has been shown to drastically improve the quality of resident-produced discharge summaries. ${ }^{16}$ Further improvements to medication lists specifically can be achieved via medication reconciliation.

\section{MEDICATION RECONCILIATION}

As the number of professionals involved in the care of one patient increases, medication information becomes increasingly fragmented among multiple informal, paper, and electronic sources. ${ }^{17}$ Medication reconciliation refers to the comparison of lists of active medications drawn up at different points in a patient's care for the purpose of detecting and correcting discrepancies. ${ }^{14}$ Sources of information include patient interview, collateral history from family members, handwritten or pharmacist-generated lists, previous lists documented in a patient's medical record, and a patient's physical medications brought to the point of care. Though known to greatly decrease medication inconsistencies and omissions, ${ }^{18}$ medication reconciliation is a laborious and technically challenging task.

Physician education has been shown to improve the quality of medication reconciliation, as have targeted patient education and empowerment; however, asking patients to bring their medications to medication review sessions (so-called brown bagging) has not worked as well. ${ }^{19}$ Fortunately, some computerised solutions have emerged to improve and streamline the process.

\section{COMPUTERIZATION AND TECHNOLOGY}

In recent years, the introduction of medication reconciliation though electronic medical records (EMRs) has given health care providers an opportunity to decrease peridischarge medication errors. In addition to compelling physicians to scrutinize admission medication lists when formulating discharge plans, electronic systems have many theoretical benefits, including guaranteed legibility, ${ }^{17}$ standardized documentation production, automatic flagging of discrepancies, and higher-fidelity communication. ${ }^{20}$ Accordingly, a randomized controlled trial involving 322 internal medicine inpatients found that an integrated electronic medication reconciliation and order entry application reduced the number of potentially harmful medication discrepancies compared to using traditional methods. ${ }^{20}$ Computerization also improves the overall quality of discharge summaries by standardizing their format and content. Electronic discharge summaries have been shown to be more understandable and lucid than their dictated counterparts. ${ }^{21}$ Email can be as effective as fax and more effective than standard mail or hand delivery in ensuring that primary care physicians received discharge summaries in a timely manner. ${ }^{17}$

Unfortunately, while existing forms of electronic systems tend to integrate well with medical products included in formulary medications that have been specifically programmed into them, other products, including herbals and supplements, are handled less ef- fectively. ${ }^{17}$ Electronic systems can also be technically difficult to implement and use ${ }^{20}$ or simply less preferred than existing methods,$^{17}$ thus decreasing physician and institution buy-in and compliance. Even with full implementation, certain types of errors still occur. For example, while computerized medication reconciliation significantly decreases erroneous inclusions of medications and unintended changes to dose, mistakes such as errors of omission are more difficult to avoid. ${ }^{22}$ Use of electronic medical records also risks encouraging counterproductive behaviours such as copying and pasting information or attaching test results indiscriminately. ${ }^{23}$

\section{EMERGING TECHNOLOGY: NEAR-FIELD COMMUNICATION}

Near-field communication (NFC) utilises short-wave radio to transfer information between devices within $10 \mathrm{~cm}$ of each other. Advantages of NFC over contemporary technologies like radio-frequency identification (RFID) or Bluetooth include its short range (and associated privacy) and negligible need for setup. ${ }^{24}$

Medical information can be stored on a small passive NFC-compatible object, then read by a powered terminal. Like a barcode, the storage device does not need to be powered, increasing portability and decreasing cost. Indeed, NFC devices shaped like small stickers or credit cards are currently available for around $\$ 1$ apiece $^{25}$ and the expectation is that future products will be even more compact and affordable. Stored information can be accessed or edited with a tap on a machine, similar to how instant payment systems currently function.

Imagine a patient arriving for a postdischarge primary care appointment with a medication list stored on an NFC-enabled sticker attached to the health card. The physician taps the card on a terminal and uses the stored information to update the patient's medical record. The EMR system draws attention to the medications that have been modified, which the physician and patient discuss. At the end of the appointment, the physician makes several medication changes. The EMR software prints prescriptions and updates the patient record. With another tap, the physician updates the medication list on the NFC sticker. This up-to-date list is returned to the patient and will be available at the next healthcare encounter, whether it be in the same office, an emergency department, an inpatient ward, or elsewhere.

This approach consolidates information into a single source that is kept with the patient and guarantees timely delivery of health information between practitioners. It also avoids the characteristic problems of having multiple medication lists that are stored in separate places and asynchronously maintained and updated. Widespread implementation will take time and resources, but the benefits described above strongly suggest it is worth the effort.

\section{CONCLUSION}

Medication errors are a major preventable cause of hospital readmissions. Medication reconciliation and computerized order entry and documentation have already decreased medication error rates. Current best practices in medication reconciliation involve pooling information from a variety of sources, but this painstaking 
process can seldom be fully accomplished, especially given the resource constraints of modern healthcare. The advent of NFC applications in health informatics may bring us closer to the elusive "one source of truth": a single accurate medication list that can be communicated efficiently and accurately between physicians.

\section{REFERENCES}

1. Canadian Institute for Health Information. All-cause readmission to acute care and return to the emergency department [Internet]. Canadian Institute for Health Information; 2012 Jun 14 [cited 2015 Dec 3]. Available from: https://secure.cihi.ca/estore/productFamily.htm?pf=PFC1823\&lang $=$ en $\&$ media $=0$

2. Elixhauser A, Steiner C. Healthcare cost and utilization project: readmissions to U.S. hospitals by diagnosis [Internet]. Agency for Healthcare Research and Quality; 2013 Apr [cited 2015 Dec 3]. Available from: http://www.hcup-us.ahrq.gov/reports/statbriefs/sb153.pdf

3. Lavenberg JG, Leas B, Umscheid CA, et al. Assessing preventability in the quest to reduce hospital readmissions. J Hosp Med. 2014 Sep;9(9):598-603.

4. PerryUndem Research \& Communications. The revolving door: a report on U.S. hospital readmissions [Internet]. The Dartmouth Institute for Health Policy and Clinical Practice, Robert Wood Johnson Foundation; $2013 \mathrm{Feb}$ [cited 2015 Dec 3]. Available from: http://www. dartmouthatlas.org/pages/readmissions2013

5. Schnipper JL, Kirwin JL, Cotugno MC, et al. Role of pharmacist counseling in preventing adverse drug events after hospitalization. Arch Intern Med. 2006 Mar;166(5):565-71.

6. Forster AJ, Murff HJ, Peterson JF, et al. The incidence and severity of adverse events affecting patients after discharge from the hospital. Ann Intern Med. 2003 Feb;138(3):161-7.

7. Bates DW, Boyle DL, Vander Vliet MB, et al. Relationship between medication errors and adverse drug events. J Gen Intern Med. 1995 Apr;10(4):199-205.

8. Lehnbom EC, Stewart MJ, Manias E, et al. Impact of medication reconciliation and review on clinical outcomes. Ann Pharmacother. 2014 Oct;48(10):1298-312.

9. Grimes TC, Duggan CA, Delaney TP, et al. Medication details documented on hospital discharge: cross-sectional observational study of factors associated with medication non-reconciliation. Br J Clin Pharmacol. 2011 Mar;71(3):449-57.

10. Jack BW, Chetty VK, Anthony D, et al. A reengineered hospital discharge program to decrease rehospitalization: a randomized trial. Ann Intern Med. 2009 Feb;150(3):178-87.

11. Pippins JR, Gandhi TK, Hamann C, et al. Classifying and predicting errors of inpatient medication reconciliation. J Gen Intern Med. 2008 Sep;23(9):1414-22.

12. Tam VC, Knowles SR, Cornish PL, et al. Frequency, type and clinical importance of medication history errors at admission to hospital: a systematic review. CMAJ. 2005 Aug;173(5):510-5.

13. Mixon AS, Myers AP, Leak CL, et al. Characteristics associated with postdischarge medication errors. Mayo Clin Proc. 2014 Aug;89(8):1042-51.

14. Kripalani S, Jackson AT, Schnipper JL, et al. Promoting effective transitions of care at hospital discharge: a review of key issues for hospitalists. J Hosp Med. 2007 Sep;2(5):314-23.

15. Hamel MB, Drazen JM, Epstein AM. The growth of hospitalists and the changing face of primary care. N Engl J Med. 2009 Mar;360(11):1141-3.
16. Key-Solle M, Paulk E, Bradford K, et al. Improving the quality of discharge communication with an educational intervention. Pediatrics. 2010 Oct;126(4):734-9.

17. Chen Y, Brennan N, Magrabi F. Is email an effective method for hospital discharge communication? A randomized controlled trial to examine delivery of computer-generated discharge summaries by email, fax, post and patient hand delivery. Int J Med Inform. 2010 Mar;79(3):167-72.

18. Nickerson A, MacKinnon NJ, Roberts N, et al. Drug-therapy problems, inconsistencies and omissions identified during a medication reconciliation and seamless care service. Healthc Q. 2005;8 Spec No:65-72.

19. Sarzynski EM, Luz CC, Rios-Bedoya CF, et al. Considerations for using the 'brown bag' strategy to reconcile medications during routine outpatient office visits. Qual Prim Care. 2014;22(4):177-87.

20. Schnipper JL, Hamann C, Ndumele CD, et al. Effect of an electronic medication reconciliation application and process redesign on potential adverse drug events: a cluster-randomized trial. Arch Intern Med. 2009 Apr;169(8):771-80

21. O'Leary KJ, Liebovitz DM, Feinglass J, et al. Creating a better discharge summary: improvement in quality and timeliness using an electronic discharge summary. J Hosp Med. 2009 Apr;4(4):219-25.

22. Allison GM, Weigel B, Holcroft C. Does electronic medication reconciliation at hospital discharge decrease prescription medication errors? Int J Health Care Qual Assur. 2015;28(6):564-73.

23. Hartzband P, Groopman J. Off the record: avoiding the pitfalls of going electronic. N Engl J Med. 2008 Apr;358(16):1656-8.

24. Ortiz CE. An introduction to near-field communication and the contactless communication API [Internet]. Oracle; 2008 Jun [cited 2016 Jan 9]. Available from: http://www.oracle.com/technetwork/articles/ javame/nfc-140183.html

25. Microsoft Devices Team. Where to buy NFC tags and how to edit them [Internet]. Windows Blog; 2013 Jan 2 [cited 2016 May 16]. Available from: https://blogs.windows.com/devices/2013/01/02/where-to-buynfc-tags-and-how-to-edit-them 